\title{
Analysis of a Ferroresonant Circuit With Chaotic Behavior by Means of a Chua-Type Magnetization Model
}

\author{
H. Endo, I. Marinova, S. Hayano, and Y. Saito, Member, IEEE
}

\begin{abstract}
This paper studies the chaotic behavior of a series ferroresonant circuit. At first, we derive a state variable equation containing a nonlinear hysteretic circuit element represented by a Chua-type magnetization model. Second, the backward Euler method with automatic modification gives transient response of the state variable equation. The characteristic values of the state transition matrix are calculated in each calculation step of Euler method. Finally, it is clarified that the characteristic values trace on the same loci although the chaotic phenomenon is exhibiting.
\end{abstract}

Index Terms-Chaotic phenomenon, Chua-type magnetization model, nonlinear calculation, series ferroresonant circuit.

\section{INTRODUCTION}

W ITH THE MODERN ferromagnetic materials, various types of electrical apparatus have been developed. Using its nonlinear magnetization characteristics, it is possible to work out constant current/voltage power supplies in heavy industry use.

To clarify the mechanism of constant current/voltage power supplies precisely, this paper carries out a series ferroresonant circuit analysis taking into account the magnetic hysteresis, saturation, aftereffects, and frequency dependences of ferromagnetic material properties [1]-[6]. To analyze the internal system stability, the characteristic values of the state variable equation of the ferroresonant circuit are calculated in each calculation period. It reveals that the changes of characteristic values have no hysteretic properties, even though chaotic phenomenon is exhibiting.

\section{Ferroresonant Circuit Analysis}

\section{A. Chua-Type Magnetization Model}

To carry out the calculation of ferroresonant circuit behavior, we employ a Chua-type magnetization model, which represents the dynamic constitutive relation between magnetic field $H(\mathrm{~A} / \mathrm{m})$ and flux density $B(\mathrm{~T})$

$$
H+\frac{\mu_{r}}{s} \frac{d H}{d t}=\frac{1}{\mu} B+\frac{1}{s} \frac{d B}{d t}
$$

Manuscript received January 16, 2003.

H. Endo, S. Hayano, and Y. Saito are with the Graduate School of Engineering, Hosei University, Tokyo 184-8584, Japan (e-mail: endo@ saitolab.com).

I. Marinova is with the Department of Electrical Apparatus, Technical University of Sofia, Sofia 1756, Bulgaria (e-mail: iliana@tu-sofia.bg).

Digital Object Identifier 10.1109/TMAG.2003.816472

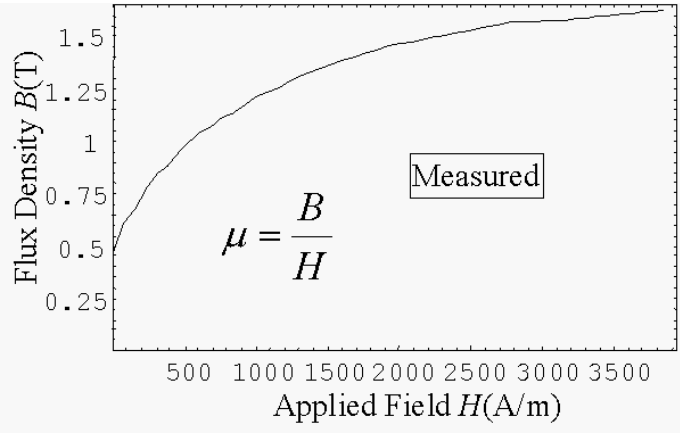

(a)

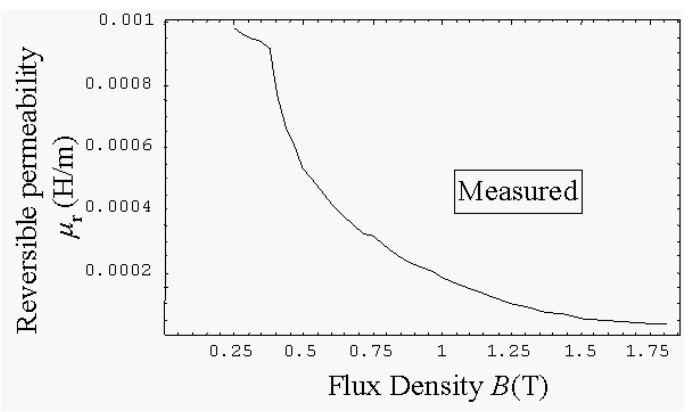

(b)

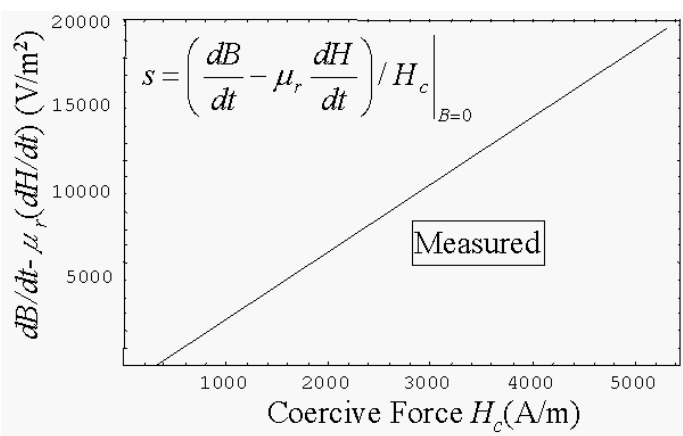

(c)

Fig. 1. Parameters of Chua-type magnetization model (measured: soft iron). (a) Permeability $\mu$. (b) Reversible permeability $\mu_{r}$. (c) Hysteresis parameter $s$.

where $\mu, \mu_{r}$, and $s$ denote permeability $(\mathrm{H} / \mathrm{m})$, reversible permeability $(\mathrm{H} / \mathrm{m})$, and hysteresis parameter $(\Omega / \mathrm{m})$, respectively [3], [5]. Fig. 1 shows the typical curves giving these parameters for soft iron. A significant feature of these parameters is that they are determined independently to the past magnetization histories. The usefulness of this model has been verified by various applications of ferromagnetic materials [6]-[9]. 


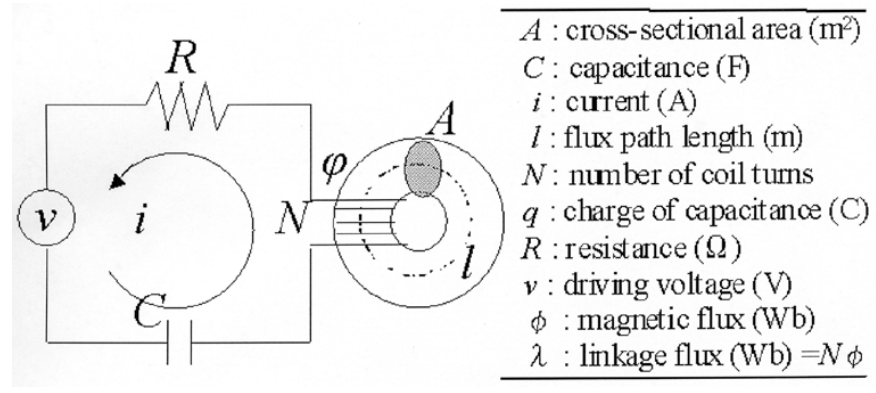

Fig. 2. Series ferroresonant circuit.

\section{B. Circuit Equation of Series Ferroresonant Circuit}

Consider a series ferroresonant circuit, shown in Fig. 2. At first, the line integral of (1) along with flux path $l$ yields the relation between the current $i$ and linkage flux $\lambda$

$$
N i+\frac{\mu_{r}}{s} N \frac{d i}{d t}=\frac{l}{\mu A N} \lambda+\frac{l}{s A N} \frac{d \lambda}{d t} .
$$

Moreover, the relation between the driving voltage source $v$ and current $i$ is given by

$$
i=\frac{1}{R}\left(v-\frac{1}{C} q-\frac{d \lambda}{d t}\right) .
$$

Second, substituting (2) into (3) yields the state (4) and (5)

$$
\begin{gathered}
\frac{\mu_{r}}{s} \frac{d^{2} \lambda}{d t^{2}}+\left(1+\frac{l R}{s A N^{2}}-\frac{\mu_{r}}{s R C}\right) \frac{d \lambda}{d t}+\frac{l R}{\mu A N^{2}} \lambda \\
+\frac{1}{C}\left(1-\frac{\mu_{r}}{s R C}\right) q=\frac{\mu_{r}}{s} \frac{d v}{d t}+\left(1-\frac{\mu_{r}}{s R C}\right) v \\
\frac{d q}{d t}=-\frac{1}{R}\left(\frac{d \lambda}{d t}+\frac{1}{C} q-v\right) .
\end{gathered}
$$

Finally, a state variable equation having $3 \times 3$ matrix $a$ is derived from (4) and (5)

$$
\frac{d}{d t}\left(\begin{array}{c}
\lambda \\
\frac{d \lambda}{d t} \\
q
\end{array}\right)=\left(\begin{array}{ccc}
0 & 1 & 0 \\
a_{21} & a_{22} & a_{23} \\
0 & a_{32} & a_{33}
\end{array}\right)\left(\begin{array}{c}
\lambda \\
\frac{d \lambda}{d t} \\
q
\end{array}\right)+\left(\begin{array}{c}
0 \\
u_{2} \\
u_{3}
\end{array}\right)
$$

or

$$
\frac{d}{d t} \mathbf{x}=a \mathbf{x}+\mathbf{b}
$$

where the elements $a_{21}, a_{22}, \ldots, u_{2}$, and $u_{3}$ are determined by (4) and (5).

\section{Calculation Procedure}

The backward Euler strategy to numerical solution of (7) performs a transient analysis of ferroresonant circuit. The calculation compares two solutions in each calculation period. One is calculated with time step width $\Delta t(\mathrm{~s})$, and the other is with $\Delta t / 2$. Evaluate the difference between these two solutions, then the solution of the period is determined. If the difference is greater than a criterion $\varepsilon$ listed in Table I, then the same period is recalculated with the modified time step width $\Delta t=\Delta t / 2$. The iteration with this modification is carried out when the criterion is satisfied.

In the iterative calculation, the nonlinear parameters, shown in Fig. 1, $\mu$ and $\mu_{r}$ are treated as functions of flux density $B$, and $s$ as a function of $d B / d t$ [7].
TABLE I

CONSTANTS FOR ANALYSIS OF FERRORESONANT CIRCUIT

\begin{tabular}{ll}
\hline$\mu:$ permeability $(\mathrm{H} / \mathrm{m})$ & Fig.1(a) \\
$\mu_{r}:$ reversible permeability $(\mathrm{H} / \mathrm{m})$ & Fig.1(b) \\
$s:$ hysteresis parameter $(\Omega / \mathrm{m})$ & Fig. $1(\mathrm{c})$ \\
$A:$ cross-sectional area $\left(\mathrm{m}^{2}\right)$ & $48.6 \times 10^{-6}$ \\
$C:$ capacitance $(\mathrm{F})$ & $22.5 \times 10^{-6}$ \\
$L:$ flux path length $(\mathrm{m})$ & $78.3 \times 10^{-3}$ \\
$N:$ number of coil turns & 180 \\
$R:$ resistance $(\Omega)$ & 1.0 \\
$\varepsilon:$ limit of discrepancy & $1.0 \times 10^{-5}$ \\
\hline
\end{tabular}

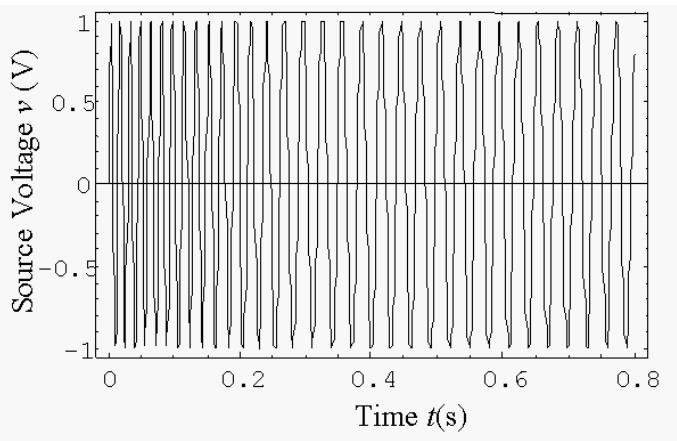

(a)

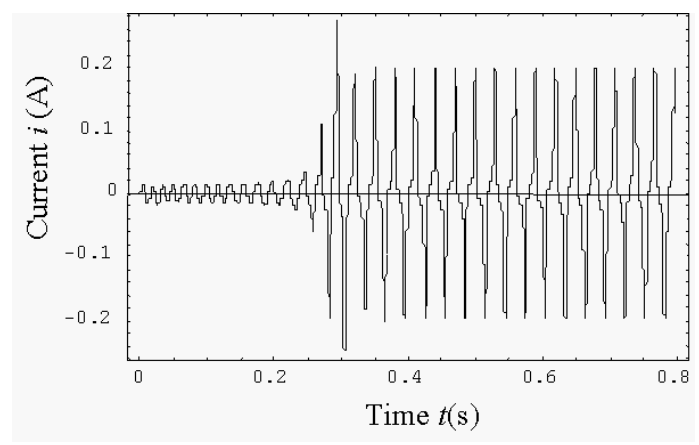

(b)

Fig. 3. Transient analysis of the ferroresonant circuit. (a) Driving voltage $v$. Frequency is decreased from 70 to $33 \mathrm{~Hz}$ until $t=0.29 \mathrm{~s}$, then fixed. (b) Calculated current $i$ versus time $t$.

\section{RESULTS AND DisCUSSIONS}

\section{A. Ferroresonant Phenomenon}

Fig. 3 shows one of the calculated results of (7) employing parameters in Table I. The experimental verification of ferroresonant circuit computation employing the Chua-type magnetization model has been reported in [6]. As shown in Fig. 3(a), the frequency of the driving voltage $v$ is decreased from 70 to $33 \mathrm{~Hz}$ until time $t=0.29 \mathrm{~s}$. Around this moment, the current $i$ drastically increases, showing the typical ferroresonant phenomenon as in Fig. 3(b). This result well corresponds to those in [6].

\section{B. Chaotic Behaviors and State of Ferroresonant System}

Fig. 4 illustrates $d i / d t$ versus $i$ obtained from Fig. 3(b), showing a chaotic property not tracing the similar locus, while the frequency of the driving voltage $v$ is fixed from $t=0.29$ s. In order to consider the state of ferroresonant system, we calculate the characteristic values of the state transition matrix $a$ in (7) to each calculation period of Euler method. The characteristic value analysis could be applied to the linear system so 


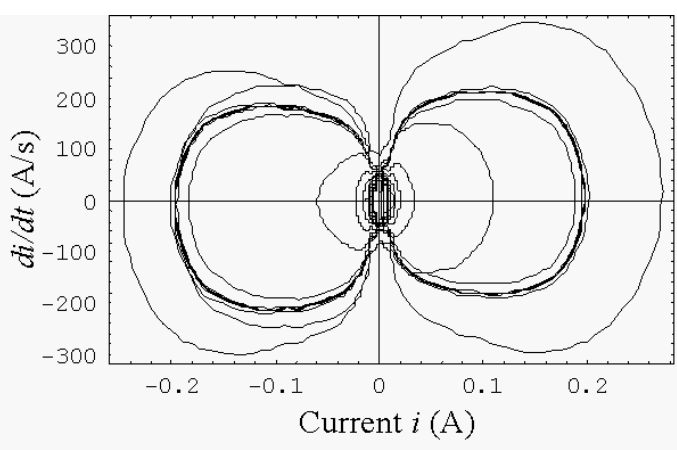

Fig. 4. Locus of $d i / d t$ versus $i$ obtained from Fig. 3(b).

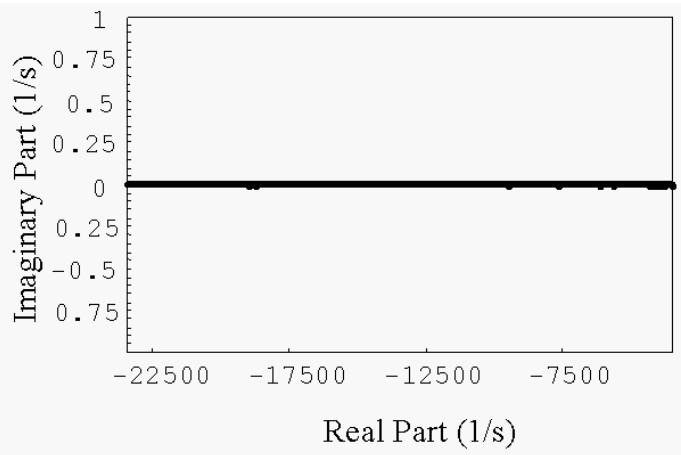

(a)

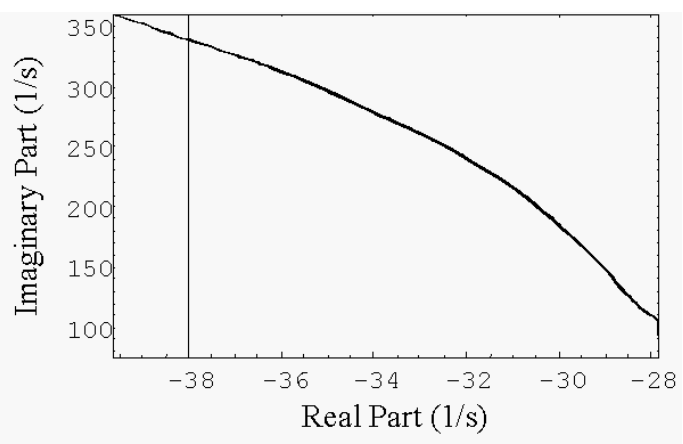

(b)

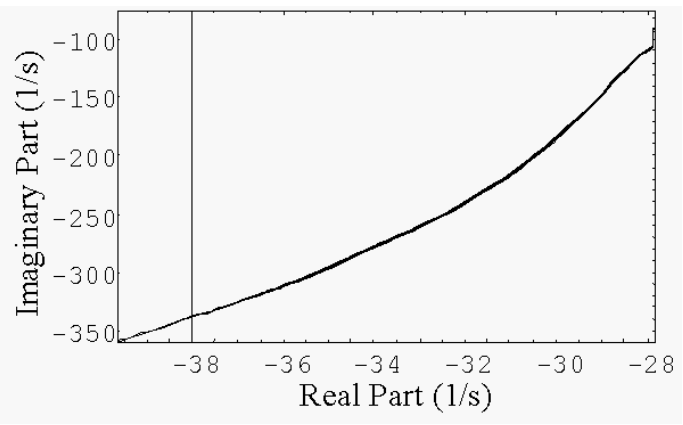

(c)

Fig. 5. Changes of characteristic values derived from matrix $a$ in (7).

that we assume this nonlinear system to be a piecewise linear system employing fine time-step widths for solving (7).

Figs. 5 and 6 show the loci of characteristic values derived from the matrices $a$ in (7). Since $a$ is $3 \times 3$ square matrix, we have three characteristic values. Even though the chaotic phenomenon is exhibiting, all of the characteristic values have a regular trajectory on the left half plane, meaning that the system

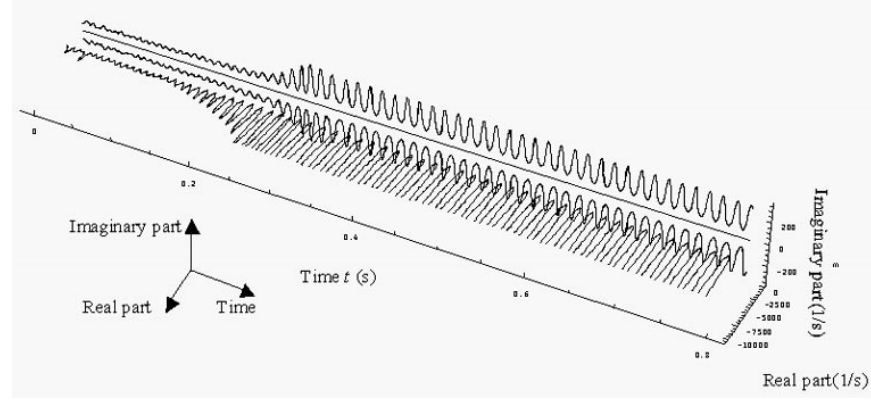

Fig. 6. Characteristic values changing of the state transition matrix $a$.

keeps in stable state. Moreover, the ferroresonant system has no hysteretic properties. Therefore, the chaotic mode is caused by the vector $\mathbf{b}$ in (7). Namely, the nonlinear coefficient $\mu_{r} / s$ on the right of (4) rouses the chaotic mode. In (1), the coefficient $\mu_{r} / s$ is a significant parameter to determine the coercive force [3], [5]. Thus, it is clarified that the change of coercive force yields the ferroresonant phenomena accompanying with chaotic flicker [8], [9].

\section{CONCLUSION}

In this paper, we have studied the chaotic phenomena of series ferroresonant circuit. We have derived the state variable equation of the circuit equations employing the Chua-type magnetization model. After that, we have carried out the transient analysis of the ferroresonant circuit. The characteristic value analysis of the state transition matrices obtained in every calculation period of Euler method has clarified that the ferroresonant system works in stable state although the chaotic flicking is accompanying. Moreover, we have clarified that this chaotic flicking is closely related to the time changing of coercive force.

This means that our approach with the Chua-type magnetization model enables us to separate the nonlinear system into stable and instable terms in magnetization processes. This is because the parameters of the Chua-type magnetization model are measured independently to the past magnetization histories.

\section{REFERENCES}

[1] F. Liorzou, B. Phelps, and D. L. Atherton, "Macroscopic models of magnetization," IEEE Trans. Magn., vol. 36, pp. 418-427, Mar. 2000.

[2] E. Della Torre, Magnetic Hysteresis. Piscataway, NJ: IEEE Press, 1999.

[3] A. Ivanyi, Hysteresis Models in Electromagnetic Computation. Budapest, Hungary: Akademiai Kiado, 1997.

[4] L. O. Chua and K. A. Stromsmoe, "Lumped circuit models for nonlinear inductor exhibiting hysteresis loops," IEEE Trans. Circuit Theory, vol. CT-17, pp. 564-574, Apr. 1970.

[5] Y. Saito, M. Namiki, and S. Hayano, "A magnetization model for computational magnetodynamics," J. Appl. Phys., vol. 69, no. 8, pp. 4614-4616, 1991.

[6] — "A representation of magnetization characteristics and its application to the ferroresonance circuits," J. Appl. Phys., vol. 67, no. 9, pp. 4738-4740, 1990.

[7] Y. Saito, S. Hayano, and N. Tsuya, "Experimental verification of a Chua type magnetization model," IEEE Trans. Magn., vol. MAG-25, pp. 2968-2970, July 1989.

[8] Y. Saito, K. Fukushima, S. Hayano, and N. Tsuya, "Application of a Chua-type model to the loss and skin effect calculations," IEEE Trans. Magn., vol. MAG-23, pp. 3569-3571, Sept. 1987.

[9] Y. Saito, S. Hayano, Y. Kishino, K. Fukushima, H. Nakamura, and N. Tsuya, "A representation of magnetic aftereffect," IEEE Trans. Magn., vol. MAG-22, pp. 647-649, Sept. 1986. 